\title{
History of Sports Medicine in the Netherlands
}

The second announcement of the XXIVth FIMS World Congress of Sports Medicine (Amsterdam, May 27June 1, 1990) refers to Holland as 'the cradle of sports medicine'. Good enough reason to look into the history of sports medicine in the Netherlands!

\section{Early years and 'The Federation of Bureaux'}

Sports medicine has a long history in the Netherlands. As early as 1906 Van den Berg was examining the influence of cycling on the heart. The scientific work of Buytendijk during the Olympic Games in Amsterdam in 1928 is well known. In later years a group around Jongbloed in the 1960s did basic research in the sports medical field.

At the time of Buytendijk's work, practising active sport was not so well accepted as nowadays. Physicians, especially, felt that there were high risks for the human being. On the other hand, there were doctors who could not agree with that opinion and said that healthy human beings could not harm their bodies by practising sports. They stressed that a good medical examination was necessary before one could take part. As a consequence, the first institute was established by Dr Reys in 1928 which took upon itself to examine athletes and give certificates for taking part in sports. The Olympic Games of 1928 in Amsterdam sped things up considerably: in the same year 10 more bureaux started their activities and in 1930 Professor Sleeswijk became the first president of the Federation of Bureaux for Sportmedical Examinations (Federatie van Bureaux voor Medische Sportkeuring in Nederland).

The main goal of the Federation was to 'eliminate the risks of sports activities as far as they were related to the physical state of health'. In other words, in those times sport was considered dangerous to health. At first, the percentage of those declared 'unfit for sport' was pretty high: in 1933 it rose as high as 9 per cent. Later on, the Federation underlined the health-benefits of sport and changed its aim 'to take measures to enable sportsmen to get the most benefit of physical exercise'. To ensure this, the Federation designed a scheme for physical examination and laid down rules and a protocol for various sports. Lectures on subjects such as 'the athlete's heart' and 'the pulse frequency' were already being given in the early 1930 s.

In the 1930s and the 1940s the sports organizations were hardly buying the services of the Bureaux for Sportsmedical Examinations. This changed dramatically in 1949, when Professor Jongbloed took over as president. Thanks to his very good relationship with the world of sport some sport leagues made a periodic sports medical examination obligatory. That gave a boost to the number of examinations and the Federation grew very rapidly to the point where, in 1979, its 227 institutes, or bureaux medically examined almost 300,000 athletes of whom 286,876 were certified fit for sport and 6,825 failed. This was achieved through the efforts of some 1500 doctors each devoting a few hours a week to bureau work. The vast majority of sport organizations in the Netherlands then required this certificate from their members before they are allowed to take part in competitive sports. It had to be renewed after one, two or more years depending on the age of the sportsman.

As one might expect, this increase in numbers affected the quality of work, which was often done for free and always in the evenings, on top of normal practice. Too many sportsmen in too little time meant hasty glances and in some cases sloppy administration. One can truly state that the success of the Federation in due course irreversibly gave way to the decline of the obligatory sports medical examination.

The early work of the Federation had been almost exclusively focussed on a very basic sports medical examination of all those practising competitive sports. This one-sided attention of the Federation to preventive aspects led to the neglect of curative and scientific aspects. Moreover, there arose a necessity for the latter two at the beginning of the sixties, when sports developed rapidly and more and more top results were aimed at. As a consequence of this, the Royal Netherlands Football Association (K.N.V.B.) founded in 1964 a sports medical department which, together with preventive sports medicine, paid more attention to curative medicine.

Within other organizations there also arose a need for sports medical guidance and knowledge, especially when the results of Dutch athletes at the 1960 Olympics were very disappointing. The Netherlands Sports Federation (NSF) organized the first additional courses in sports medicine. The physicians who followed this course and various other doctors concerned with guidance of Olympic athletes and soccer players, founded the Netherlands Association of Sports Medicine in 1965 with Professor Jongh as its first president.

\section{Netherlands Association of Sports Medicine}

For the first ten years of its existence the Association concentrated on sports medical guidance of top athletes, scientific research and additional education of physicians in sports medicine. At its tenth anniversary, the Association realized that the image of sports medicine was still below the required standard. Too often sports medicine was associated with malpractice (for instance doping) or its quality was judged by the 


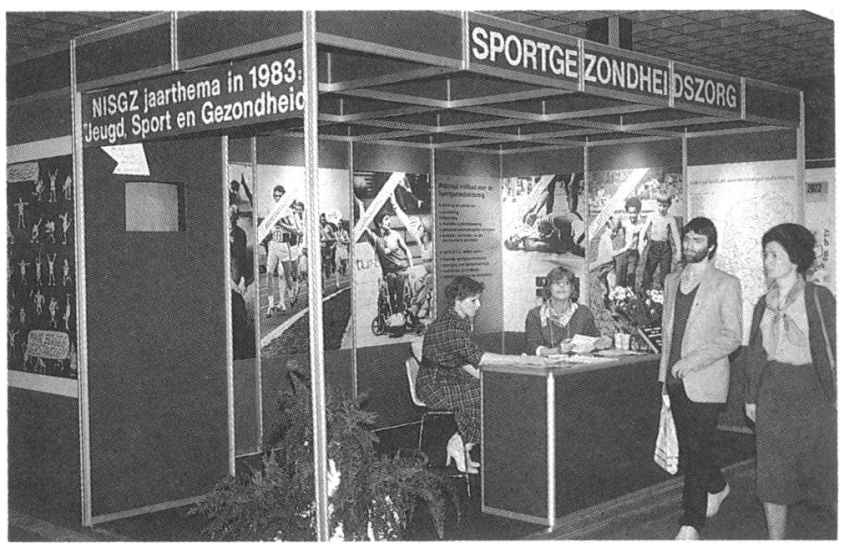

time a top athlete took to recover from injuries and no importance was attached to the seriousness of the injury.

The board of the Association then stated that sports medicine had to develop a first rate sports health care, accessible to all. 'Sports health care' is understood to mean the care and protection of the physical and psycho-social health of sports participants. An extensive plan was produced in 1975 and offered to the Minister of Health. To put it plainly, no great results were expected but by coincidence the starting point of a very rapid development of sports health care in the Netherlands was made. In the same year, 1975, the Totosystem was altered and the Lotto-Toto was introduced. On the board of the Lotto-Toto a place was made for a representative of the sports medical world and so influence was gained upon the spending of money.

The first result was the foundation of the BSGA, which is the combined executive bureau of the Association and the Federation. Hereby an opportunity was given to achieve centrally organized and directed sports health care system.

At the same time the National Sports Federation had a sports medical department added to it. In fact, there was a lack only of centres which could advise top sportsmen or those athletes who needed special advice for which a good sports medical knowledge is essential. This gap was filled by providing sports medical consultation centres (SMA), which have a regional function.

\section{The SMAs}

A sports medical consultation centre is an institute where there is a concentration of sports medical and sports physiotherapeutic knowledge. On the basis of this knowledge physicians and physiotherapists can give guidance to sportsmen at any level as well as to those with specific problems in relation to sports. They can also give expert advice on sports medical problems in general.

Although these sports medical consultation centres (SMA's) in principal have a mainly preventive task, they are slightly curative and at the same time well equipped for performing exercise testing. They are evenly spread throughout Holland. Because their continued existence depends on first rate medical

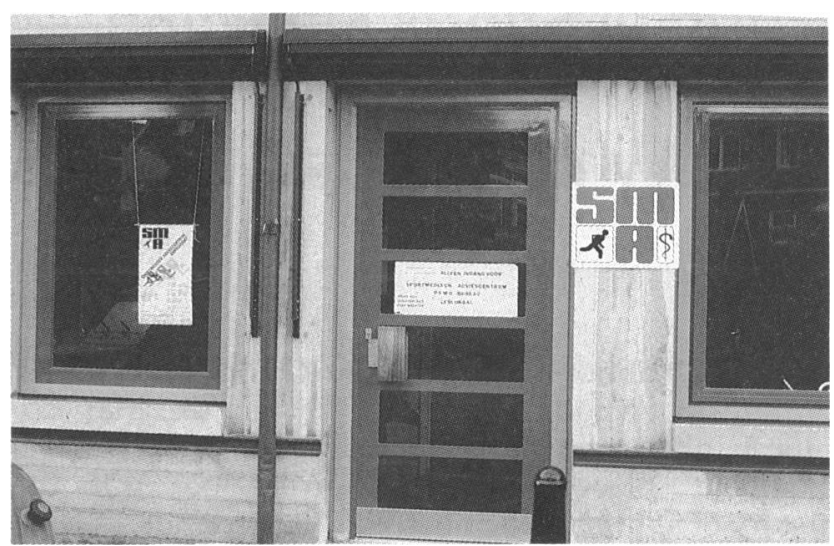

standards, the Association has a standards committee and fulfilment of standards is controlled by the accreditation committee, which is an independent body, body, on which medical and public authorities are represented.

A rapidly rising budget enabled the Association and Federation to fulfill their basic tasks:

- Education

- Preventive sports medicine

- Organization

- Information

- Promotion

- Scientific research promotion

- Journal.

Education (see article on NISHC, p.222)

- Training during basic medical curriculum

- Additional education

- Specialization SOS.

In close cooperation with other medical organizations the Association concerned set up a four year sports medical specialization. For this goal a foundation was formed, the so-called SOS, in which the Association cooperates with the Netherlands Heart Foundation, the Netherlands Sports Federation, the Royal Netherlands Football Association and the Royal Netherlands Medical Association.

In the early seventies things changed rapidly. The sports organizations began to show interest in the practice of sports medicine, financial support from Toto-Lotto became a reality and organizations like the Netherlands Heart Foundation embraced sports medicine. Even the government looked kindly upon it. To a great extent, this was the work of 'the last of the pioneers': Professor Mosterd, who started his career as the first full time sports physician (in professional soccer) and who played an important role in the development of sports medicine in the seventies and the eighties. In 1988 he became the first professor in sports medicine at the University of Utrecht. In the meantime he presided over the Netherlands Association of Sports Medicine; he still presides over the National Institute for Sports Health Care and now also the Foundation for the 1990 FIMS World Congress of Sports Medicine.

The NASM published in 1976 a policy view, the 
'Future of Sports Medicine in the Netherlands'. The main items were:

- To create new possibilities for examination and guidance

- To introduce nationwide accessible sport medical units

- To adapt to the specific needs of sportsmen

- To stimulate education and information.

This policy view is certainly a landmark in the history of Dutch sports medicine. It led to a chain of sports medical advice centres, where sportsmen can get extensive examination and sport-specific advice. It also meant more education as the need for sports medical experts was great. The NASM expanded the applied course in sports medicine and established a four year education for specialists in sports medicine. In 1979 the first sports medical specialist graduated.

Also in 1980, the Federation and Association were merged and a regional network instituted based on groupings of one SMA and five or six bureaux.

\section{Foundation of National Institute for Sports Health Care (NISHC)}

National Institute for Sports Health Care was launched in 1982. The object of this institute is 'to raise sports health care in the Netherlands to a higher level and to make it accessible for all sportsmen'.

This institute rapidily became a well known authority and undertook some important activities. In spite of a lot of effort the NISHC has not succeeded in keeping a basic sports medical examination alive; the sports organizations in Holland have rejected a sound protocol for about 25 guilders (approximately £7), the 'periodic sports medical examination'. Sports medical examinations now take place at the 40 sports medical advice centres. They see about 10,000 sportsmen a year out of a total population of more than 4 million sportsmen.

The NISHC publishes many sports medical brochures and books and has organized congresses and campaigns on a large scale, often linked with annual themes like 'Chronic diseases and sports', 'Doping', 'Prevention'.

In 1986 sports medicine was officially registered as a specialism. At this moment some 25 sports medical specialists have completed this training. The traditional medical world has developed more interest in sports medicine: the Royal Netherlands Society for the Promotion of Medicine (the largest professional society) held its 1982 annual congress on sports medicine and every year school doctors follow retraining courses in sports medicine. Broader interest can also be measured by the NASM periodical Geneeskunde en Sport, which has a circulation of about 3,000 (the NASM has 500 members). Leading articles have been published, which often led to general publicity in papers, on radio or television.

The latest development is the Janus Jongbloed $\mathrm{Re}$ search Centre, a sports medical scientific institute linked to the University of Utrecht with Professor Mostered as its head. The Dutch are very active in international contacts, especially within FIMS. Dr Hermans is a member of the FIMS Board. The Netherlands is also active in the Northwest Europe Chapter of FIMS.

And now the 1990 FIMS World Congress of Sports Medicine is the latest challenge for the Netherlands. The Dutch certainly will see to it that they maintain their high reputation! 